\title{
Germanica
}

\section{Entretien avec Robert Menasse}

Robert MENASSE „Wir leben in einem 'nicht mehr- noch nicht'- Zustand“

Julie Anne Demel

\section{(2) OpenEdition}

\section{Journals}

Édition électronique

URL : http://journals.openedition.org/germanica/2918

DOI : 10.4000/germanica.2918

ISSN : 2107-0784

Éditeur

Université de Lille

\section{Édition imprimée}

Date de publication : 30 septembre 2015

Pagination : 141-146

ISBN : 9782913857353

ISSN : 0984-2632

Référence électronique

Julie Anne Demel, « Entretien avec Robert Menasse », Germanica [Online], 56 | 2015, Online erschienen am: 30 September 2017, abgerufen am 06 Oktober 2020. URL : http://journals.openedition.org/ germanica/2918; DOI : https://doi.org/10.4000/germanica.2918

(c) Tous droits réservés 


\title{
Entretien avec Robert Menasse 1
}

\author{
Robert MENASSE \\ „Wir leben in einem 'nicht mehr- noch nicht'- Zustand“
}

(Nach einem Telefongespräch mit Julie Anne Demel)

Der Titel Der europäische Landbote verweist auf den Büchnertext Der hessische Landbote. Büchner richtet seinen Text an die Bauern und die Bürger des Landes Hessen. An wen richtet sich Der europäische Landbote? Ist dieses Buch nur für das österreichische Publikum gedacht?

R. M. : Der Text richtet sich nicht nur an das gesamte deutschsprachige Publikum, sondern an alle mündigen Bürger Europas. Der Landbote erscheint demnächst im Frühling in französischer Sprache ${ }^{2}$.

Büchner richtet sich gegen die feudale Willkür der Kleinstaaterei. Heute gilt es die Nationalstaaten zu überwinden und der Appell richtet sich gegen die Willkür der Konzerne.

Der Büchner Text hat unmittelbar die Revolution von 1848 beeinflusst. Wollen Sie mit Ihrem Text einen Flächenbrand in Europa auslösen?

R. M. : Das Buch Der europäische Landbote will vor allem die europäische Idee rekonstruieren. Das europäische Projekt ist eigentlich eine europäische Revolution: Es ist revolutionär, ein Europa ohne die Nationalstaaten zu denken. Historisch gab es schon

1. - La rédaction tient à exprimer ses remerciements à Robert Menasse pour l'entretien qu'il a accordé à Julie Anne Demel dans le cadre de ce numéro.

2. - Robert Menasse, Un messager pour l'Europe. Plaidoyer contre les nationalismes, traduit par Dominique Venard, Paris, Buchet-Chastel, 2015. 
Zusammenschlüsse, ohne an die Nationalstaaten zu denken. Die heutige Welt ist fix in der Globalisierung verankert. Alle Prozesse seien es Finanzströme, Investitionen, die Kommunikation oder das Internet - sind transnationale Gegebenheiten. Europa versucht diesen entgegenzuwirken.

Durch das Buch gilt es die politische Idee der Gründerväter zu rekonstruieren, aber auch den Prozess zu beschreiben, was in Brüssel tatsächlich passiert. Wir leben in einem ,nicht mehr- noch nicht“Zustand. Wir befinden uns in einer aktuellen Zwischenstation. Durch die Krise funktionieren die herkömmlichen Mechanismen nicht mehr, aber die nachnationale Entwicklung ist noch nicht fortgeschritten. Das ist ein Widerspruch. Es gilt die herkömmliche Souveränität zu überwinden.

Die Figur des Landboten gibt es heutzutage nicht mehr. Früher war es eine zuverlässige Möglichkeit von einem Ort zum anderen Ort Botschaften zu bringen. Auch druckt man heute keine Flugschriften mehr. Heute gibt es eine Hyperinflation an Nachrichten und Twitterund Facebook-Meldungen. Dennoch hat man das Gefühl, dass es trotz der Hyperinformation ein Kommunikationsdefizit gibt.

R M: Es herrscht tatsächlich ein Kommunikationsdefizit vor. Die nationalen Medien filtern die Informationen für ihre Leserschaft heraus. Man erfährt nur eine partielle Information, zum Beispiel: was kostet uns dies und jenes. Es steht nur das nationale Interesse im Vordergrund.

Die Menschen haben zu wenig Interesse. Der normale österreichische Bürger kennt sich auch nicht im österreichischen System aus: Er fragt sich nicht, was in der österreichischen Verfassung steht. Er weiß auch nicht, wer die Gesetze beschließt. Niemand weiß, wie die Landeskonferenz funktioniert. Niemand kann das österreichische System erklären. Man begnügt sich mit den vorgefundenen Verhältnissen. Es wird vor allem eine Ausblendung der Probleme der Nationalstaaten betrieben.

$\mathrm{Zu}$ betonen ist die doppeldeutige Haltung des Bundeskanzlers Faymann, was Europa betrifft. In Österreich zeigt er sich eurokritisch aber im Europäischen Rat beschließt er die Gesetzgebung mit. Nach innen prangert man den Sündenbock an, nach außen trägt man weitreichende Entscheidungen mit.

Wer sich informieren will, hat genug Möglichkeiten. Die Kronen Zeitung prangert die steigende Kriminalität an. Aber nach den politischen Entscheidungen hat keiner mehr ein Bedürfnis. Es gibt keine Debatte, wie man die Krise lösen kann. In der Presse steht nur, welche Entscheidungen der Präsident durchsetzen konnte. 
Aber es gibt auch andere Kanäle wie zum Beispiel die Social Media. Da besteht auch die Gefahr, dass Gerüchte statt Fakten kursieren.

Bezüglich des Begriffes „Bürger“ entwerfen sie eine Dialektik des Begriffes. Heutzutage gibt es nur entweder ,Wutbürger “, „Mutbürger“ oder „Präfixbürger“. Früher hatte der Bürger Rechte und Pflichten. Heutzutage beharrt der Bürger auf seinen Rechten, aber nimmt kaum seine Pflichten in Anspruch. Fehlt es an einer Zivilgesellschaft?

R. M. : Der deutsche Bürger unterscheidet sich vom französischen Bürger. In Frankreich unterscheidet man wieder unter „citoyen“ und „bourgeois“. Der deutsche Bürger definiert sich durch das Wahlrecht. Das Wort Bürger ist im Deutschen jetzt zum Teil negativ behaftet: man denkt an den Kleinbürger oder Spießbürger.

Der Verfassungsrechtler Hans Kelsen hat gemeint, dass der Demokratie ein gebildeter ,citoyen“ vorausgeht. Das Problem ist, dass alle Demokratiemodelle Mehrheitsdemokratiemodelle sind. So meint Hans Kelsen weiter, dass die Demokratie dem Schutz der Minderheit dient und eine Kompromissfähigkeit mit der Mehrheit nötig ist.

Der „Wutbürger“ ist ein Ausdruck von Ressentiment. Die Bürger können nur Ressentiment empfinden und die Bevölkerung tritt immer radikaler auf, z. B. jetzt in Dresden die Demonstrationen gegen die muslimische Bevölkerung. Es ist der Ausdruck von panischen Menschen mit sozialem Abstieg.

Es muss eine transnationale und postnationale Demokratie entstehen. Die Ideen müssen demokratisch legitimiert sein. Es muss eine neue Form von Demokratie entstehen. Die herkömmlichen Begriffe wie „proletarisch“ und „citoyen“ haben ausgedient.

Das ist das Paradox unserer Zeit: Formalrechtlich ist der Leiter großer Konzerne ein Angestellter. So untersteht der Leiter des Konzerns dem Schutz der Gewerkschaft. Der Start-up Unternehmer, der geringfügig beschäftigt ist, hat Projektverträge eines Kleinunternehmers, wird aber nicht von der Gewerkschaft unterstützt. Die Gewerkschaft stützt die Starken und nicht die Schwachen in der Gesellschaft.

Meistens haben soziologische Gruppen klare Interessen. Es herrscht, wie es Marx ausgedrückt hat, ein Klassenbewusstsein vor. Heute gibt es dieses Klassenbewusstsein nicht mehr. Man weiß nur, dass man ein bedingungsloses Grundeinkommen haben will, eine entfremdete Arbeit hat, und dass es ein schlechtes Bildungssystem gibt. Es gibt einen Transformationsprozess, aber es gilt eine nachnationale Idee zu definieren. 
Sie wollen also einen supranationalen Staat?

R. M. : Nicht unbedingt! Es gibt supranationale Institutionen. Die UNO ist zum Beispiel ein solches Konstrukt und muss jeden Tag nationale Interessen der Nationalstaaten überwinden. In Europa ist diese Entwicklung stärker. Das EU-Parlament hat nicht alle Rechte, die Kommission ist keine Regierung und die Zentralbank hat nicht alle Kompetenzen.

Es gibt kein vollkommenes Demokratiemodell. In der aktuellen Konstellation wird die nationale Demokratie geschwächt, aber die Probleme nicht.

Bronislaw Geremek meinte, dass Europa die Demokratie vervollständigt und zu Ende gefuihrt hat?

R. M. : Jedes Land strebt nach einem nationalen Modell von Demokratie, das es unweigerlich verteidigt. Jedoch verteidigt jedes Land meistens nur die Basisinteressen, die da sind: Sicherheitszustand - und wirtschaftliche Interessen. Die nationalen Interessen werden von den jeweiligen Eliten vorgeprägt.

Welche Rolle kann die Literatur spielen?

R. M. : Die Schriftsteller haben weitblickend schon gesehen, dass die Staaten nur im Frieden leben können, wenn die nationalen Interessen überwunden sind.

Victor Hugo hat schon 1847 vor dem überspitzen Nationalismus gewarnt. Er vertrat die Ansicht, dass die Französische Revolution die französischen Provinzen zusammengeschweißt hätte, ohne ihre Eigenart zu zerstören. Die deutsche Nation war damals eine Zusammensetzung von über 40 Kleinstaaten.

Stefan Zweig schreibt 1913: Wir stehen vor einem Todeskampf der Nationen. Der Nationalismus ist das Grundübel, um ein gemeinsames Europa zu verhindern. 1914 folgte dann erneut ein 30 jähriger Krieg in Europa, der bis 1945 dauerte. Nach dem Krieg musste die Gründergeneration die Feindschaften der Nationalstaaten überwinden.

Im Moment findet eine Renationalisierung statt, anstatt ein gemeinsames Europa zu schaffen. Schon Novalis hat den Traum für ein gemeinsames Europa in seiner Europa-Rede angedeutet. Der Germanist Lützeler hat sogar eine Anthologie von allen Europatexten verfasst ${ }^{3}$.

3. - Paul Michael Lützeler, Die Schriftsteller und Europa. Von der Romantik bis zur Gegenwart, München, Piper Verlag, 1992; vgl. auch ders., Kontinentalisierung - Das Europa der Schriftsteller, Bielefeld, Aisthesis Verlag, 2007. 
Träumen Sie von einem neuen Europa? Denken sie da an eine Utopie?

R. M. : Keine Utopie - Utopie heißt nirgendwo. Es gilt an die konkrete Realpolitik zu denken. Der europäische Prozess ist schon konkret. Wir leben in einem globalen Kontext, in dem es gilt, die nationale Beschränktheit und die nationalen Grenzen zu überwinden - schon allein durch den Austausch im Internet. Es gilt konkret, die Realpolitik zu gestalten und als Bürger mitzugestalten.

$(22.12 .2014)$ 
